\title{
Innover par le passé
}

\author{
Damien Hallegatte ${ }^{a}$
}

RÉSUMÉ. Le présent article avance que le dogme de l'innovation, qui implique une mise en obsolescence de plus en plus rapide des produits, pourrait se retourner contre les organisations qui pensent que les consommateurs croient encore fortement au progrès. Nonobstant les questions cruciales relatives à l'environnement, c'est peut-être le basculement de notre rapport collectif à notre passé qui pourrait sonner le glas de l'innovation, telle que nous la connaissons aujourd'hui, comme voie royale pour atteindre les objectifs des organisations. Il semble qu'au XXIe siècle, la voie de l'avenir pour les organisations se trouve en partie dans la réappropriation du passé.

ABSTRACT. In this article, I suggest that innovation dogma, which imply rendering products obsolete faster and faster, could backfire against organizations which believe that consumer still strongly believe in progress. Apart from environmental concerns, it may be the change of how we collectively relate to our past that could stop innovation being seen as the most effective way to reach organizational aims. In the $21^{\text {st }}$ century, the path to the future may partially lie in the past.

\section{Introduction}

Le phénomène n'a échappé à aucun gestionnaire. Le passé a fait un grand retour dans nos vies depuis plusieurs années. Des vieux groupes de rock comme The Rolling Stones ou Led Zeppelin, aux automobiles comme la Mini ou la Fiat 500, en passant par les produits électroniques ou de décoration au style rétro, à peu près toutes les catégories de produits ont été touchées par le phénomène rétro. On voit même des technologies obsolètes réapparaître, comme le disque vinyle et, plus récemment, la cassette audio.

Dans certains cas, l'attrait du passé est tel qu'il n'y a même plus besoin de faire preuve de créativité. Les tournées de concert de The Police en 2007 et 2008 et de Roger Waters entre 2010 et 2013, ont généré respectivement 362 et 459 millions de dollars ${ }^{1}$, alors que ces artistes se sont contentés d'interpréter des chansons créées des décennies plus tôt. De son côté, Michael Jackson, grâce au Cirque du Soleil, a produit un revenu de 371 millions de dollars entre 2011 et 2014, alors même qu'il était mort.

Le rétromarketing, qui consiste à faire revivre d'anciens produits ou d'anciennes marques, en conservant de manière permanente et explicite leur association avec le passé, est ainsi devenu une stratégie viable au XXIe siècle. En outre, il est encore plus courant de susciter la nostalgie dans une publicité, même pour des produits qui ne sont pas rétro. En effet, contrairement au rétromarketing, la nostalgie en publicité peut théoriquement s'appliquer à n'importe quel produit.

Tout ceci est susceptible de créer de la dissonance cognitive à quiconque a suivi un cours de marketing. On nous enseigne en effet que la raison d'être de cette discipline est de créer la prochaine innovation que le marché va accepter, de promouvoir la nouveauté, et ainsi de participer au progrès de la société. Or, le rétromarketing, qui est pratiqué par de nombreuses organisations, implique de ressusciter des produits, des marques, des technologies et des styles anciens, mis en obsolescence par les professionnels du marketing eux-mêmes. Et, bien que ces produits soient mis à jour techniquement, ils ne le sont pas symboliquement, par définition.

La discipline du marketing, et le monde des affaires en général, se retrouve donc en contradiction avec leur vision progressiste (Brown, 2001) en admettant implicitement que des choses appartenant au passé peuvent avoir autant de valeur, sinon plus, que des choses actuelles. En vendant des produits

\footnotetext{
a Professeur, Ph. D., Univerrsité du Québec à Chicoutimi
} 
obsolètes, le marketing, pour lequel l'innovation est un maître-mot, semble se désavouer lui-même. Manifestement, quelque chose s'est passé.

\section{Retour vers le passé}

Au moins une chose est sûre, le passé se vend désormais très bien. Si on observe des signes d'intérêt pour quelques produits rétro dès les années 1960, c'est durant les années 1990 que la courbe des ventes de ce qui appartient au passé a pris une forme exponentielle. Les produits rétro ont progressivement atteint de nombreuses catégories de produits, y compris celles résolument tournées vers l'innovation, comme l'automobile ou l'électronique, et tous les segments de marché, y compris les jeunes.

En première analyse, on peut considérer ce phénomène comme un éclectisme arbitraire ou un simple effet de mode. Mais il s'agirait là d'une interprétation confortable et simpliste, quoique séduisante puisque ne remettant pas en question le dogme de l'innovation et la croyance dans le progrès.

En deuxième analyse, on peut y voir un basculement du rapport des sociétés occidentales à leur passé récent, et plus généralement un changement de notre rapport au temps. En particulier, dans notre monde postmoderne, où les tenants de la modernité, dont la notion de progrès, sont remis en cause, l'opposition structurelle entre le passé, le présent et le futur semble voler en éclats. Mais c'est surtout le jugement de valeur de la supériorité systématique des époques suivantes sur les précédentes, lequel sous-tend la notion de progrès, si chère au modernisme, qui se retrouve remis en question. S'il est indéniable que le monde change, il n'est pas sûr que ce soit pour le mieux, en tous cas pas sur tous les plans, et certainement pas pour tout le monde.

Cette croyance en une évolution chronologique essentiellement positive a beaucoup servi les organisations qui souhaitent inciter le consommateur à acheter chaque nouveau produit. Mais aujourd'hui, les défis écologiques et démographiques sans précédent auxquels nous commençons à faire face ont nourri un scepticisme grandissant par rapport à la notion de progrès, la base idéologique de l'innovation.

Mais c'est peut-être un ras-le-bol des promesses non comblées de la société de consommation qui pourrait porter le coup le plus dur à cette croyance moderne. En effet, à en croire l'économie néolibérale, c'est le consommateur, par ses choix de consommation, qui peut changer le monde le plus efficacement. Il semble qu'il ait commencé à le faire en achetant des produits rétro et en se vautrant sans gêne dans la nostalgie. Le message envoyé peut être interprété de la manière suivante : trop d'innovation, c'est comme pas assez. Les consommateurs se sentent bousculés par la marche du progrès.

Certes, en première analyse, acheter des produits rétro est aussi bon pour l'économie qu'acheter des produits non rétro. Mais la logique sous-jacente de valorisation de ce qui est ancien pourrait conduire la masse des consommateurs à se procurer des produits usagés ou à prolonger leur durée de vie de ceux qu'ils possèdent déjà. Le phénomène rétro contient donc les germes de pratiques réellement subversives pour l'économie telle qu'on la connait.

Par ailleurs, plutôt que de voir dans le phénomène rétronostalgique une anomalie par rapport à la notion de progrès, on pourrait au contraire considérer le progrès lui-même, mais surtout le rejet du passé qu'il implique, comme une anomalie. En effet, cette notion moderne n'est portée au pinacle des valeurs du monde occidental que depuis le Siècle des lumières. Ainsi, le retour en grâce du passé après plus de trois siècles d'opprobre pourrait n'être qu'un retour à la normale.

Notons, en passant, qu'en l'instrumentalisant grossièrement, c'est peut-être l'économie de marché qui a perverti la notion de progrès, en accentuant sa dimension de rejet du passé. Il serait donc injuste de faire le procès de l'idée de progrès per se, et encore moins d'affirmer que l'aliénation de l'homme moderne vis-à-vis du passé, c'est la faute à Voltaire, ou bien c'est la faute à Rousseau.

Cependant, si notre réconciliation avec le passé apparait comme une tendance de fond, elle ne semble néanmoins aujourd'hui que partielle. Le désir des consommateurs de se replonger dans le passé récent est probablement loin d'être assouvi, puisque encore brimé par des relents modernes de jugement négatif du passé. Cela procure des occasions aux organisations qui oseront proposer et faire la promotion de produits rétro dans des catégories de produits où l'on est probablement loin 
d'avoir atteint la saturation (ex. : équipements sportifs, produits électroniques...). Dans le domaine des services, ce pourrait être un retour de l'humain.

Cependant, la manière de se relier au passé, et notamment à son passé organisationnel, est une décision stratégique qui ne saurait être laissée au hasard, ni même déléguée à une agence de publicité. En effet, les conséquences sur la perception des marques, des produits et des services que gèrent les entreprises peuvent être profondes et durables.

Afin de saisir la profondeur du phénomène, et de faire sauter les verrous psychologiques encore existants relativement au passé et à la nostalgie, il est utile de se pencher sur les raisons qui nous ont conduit à nous réconcilier, surtout à partir des années 1990, avec notre passé. Ensuite, je proposerai de distinguer deux stratégies de gestion d'une marque perçue comme appartenant au passé.

\section{La nostalgie n'est plus ce qu'elle était}

C’est peut-être en 1989 précisément que le passé a entamé son retour en force dans nos vies. À l'époque, on observait un écart d'approximativement 20 ans entre la période contemporaine et celle à laquelle on faisait référence dans les premières publicités dites nostalgiques (Havlena et Holak, 1991). Or, 1989 était l'année du vingtième anniversaire des premiers pas humains sur le sol lunaire, événement qui peut être considéré comme l'apogée de l'humanité, et du festival de Woodstock, symbole de l’idéalisme des années 1960.

Cette même année 1989 a vu aussi le début de l'effondrement du communisme en Europe, qui a mené à la disparition du choc des grandes idéologies séculières, au triomphe de la démocratie libérale et, ainsi, selon Fukuyama (1992), dans une perspective hégélienne, à la «fin de l'histoire». Cette coïncidence politicohistorique a peut-être rendu le futur en même temps inintéressant et incertain et, parallèlement, le passé rassurant et attirant.

Au même moment, les premiers bébé-boumeurs sont entrés massivement dans la quarantaine, un âge où on a atteint un certain accomplissement, et où l'on commence à regarder en arrière. Après avoir contesté l'ordre établi et avoir atteint le confort matériel, cette

Revue Organisations \& Territoires $\bullet$ Volume $27 \bullet \mathrm{N}^{\circ} \mathbf{1} \bullet 2018$ génération a recherché le confort psychologique des produits rappelant leur jeunesse.

La taille et la solvabilité de ce segment de marché l'ayant rendu particulièrement attractif, les organisations les moins obnubilées par la notion de progrès et d'innovation ont commencé à répondre à leurs désirs. C'est donc en réalité une triple coïncidence historique qui s'est produite à la fin du siècle dernier, alors que le contexte historique, politique et démographique a modifié le rapport de la société occidentale avec son passé récent.

Par conséquent, le concept même de nostalgie a suivi cette évolution (et non l'inverse). La nostalgie était considérée, en 1688, par l'inventeur du mot, le médecin suisse Johannes Hofer, comme une maladie pouvant être traitée par des purges, des saignements ou l'absorption de certaines mixtures. Il a fallu attendre l'ouvrage du sociologue Fred Davis en 1979 pour que la nostalgie soit définitivement expurgée de son aspect morbide. Mais c'est seulement à partir des années 1990 que la recherche scientifique en marketing a commencé à découvrir les avantages, pour les organisations, de la nostalgie des consommateurs.

Il est aujourd'hui solidement établi que susciter ouvertement cette émotion dans une publicité constitue une stratégie efficace, puisqu'elle influence positivement l'attitude des consommateurs envers la publicité, la marque et le produit annoncé, ainsi que l'intention d'achat (par ex., Muehling et Pascal, 2011). En effet, la nostalgie crée des émotions plus positives et évoque des images mentales plus intenses, tout en induisant des niveaux plus élevés de réflexion sur soi et d'implication envers la publicité. Ainsi, la nostalgie est une émotion souhaitable, et non un sous-produit d'une stratégie basée sur l'évocation du passé. Il s'agit même d'une émotion stratégique, en raison de ses effets positifs, qui sont de nature à renforcer le lien affectif entre l'organisation et ses clients.

En outre, les recherches en psychologie ont, depuis une dizaine d'années, montré que la nostalgie a des effets positifs sur le bien-être psychologique des individus (par ex., Wildschut et al., 2010). Ressentir cette émotion familière, à la signature affective principalement positive, remplit des fonctions existentielles, et permet notamment de faire face plus sereinement à la perspective de sa propre mort. En 
effet, être nostalgique permet de se sentir profondément connecté à un passé, par définition, heureux. En outre, cette émotion complexe déclenche une activité cognitive qui conduit à penser que la vie a valu la peine d'être vécue, et qu'elle le vaudra probablement encore.

\section{Le rétromarquage, stratégie passéiste}

Fort de cette réconciliation avec le passé et de la réhabilitation de la nostalgie, la discipline du marketing ne s'est pas contentée d'utiliser cette émotion comme ressort publicitaire. On s'est mis à vendre le passé (Brown, 2001). Le rétromarquage, ou retrobranding, cas particulier de rétromarketing où une marque du passé est relancée (Brown, Kozinets et Sherry Jr., 2003) est devenu une stratégie viable. Cependant, le rétromarquage est confondu dans la littérature scientifique et professionnelle avec la notion bien connue de revitalisation de la marque, telle que présentée, notamment, par Keller (2013).

Or, les organisations qui veulent profiter du regain d'intérêt de leurs clients pour le passé devraient pourtant bien distinguer ces stratégies, afin de faire un choix stratégique éclairé. J'ai suggéré de considérer le rétromarquage comme la revitalisation ou la relance d'une marque que l'on soubaite garder associée au passé (Hallegatte, 2014). Il ne s'agit pas de ramener une marque ou un produit dans le présent, mais bien de préserver son association avec le passé. Cette idée est révolutionnaire pour la discipline du marketing.

Dans cette perspective, on peut distinguer deux types de stratégies de marque reliées au passé. Dans les cas Burberry, Lacoste, Mountain Dew ou Harley-Davidson, par exemple (Keller, 2013), on a ramené une marque déclinante dans le présent, c'est-à-dire qu'il s'agissait de classiques stratégies de revitalisation de la marque. Mais dans le cas de la Mini, la Fiat 500, Led Zeppelin, ou Elvis Presley, par exemple, l'approche consistait au contraire, et consiste toujours, à préserver l'association explicite de la marque avec le passé, fusse-t-elle de plus en plus discrète en ce qui concerne les automobiles. Il s'agissait de stratégies de rétromarquage. De cette différence de principe découlent plusieurs autres (voir tableau 1).

\begin{tabular}{|l|l|l|}
\hline & Revitalisation de la marque & Rétromarquage \\
\hline Principe & $\begin{array}{l}\text { Ramener une marque dans le } \\
\text { présent }\end{array}$ & $\begin{array}{l}\text { Préserver l'association de la marque } \\
\text { avec le passé }\end{array}$ \\
\hline Référence historique & Histoire de la marque & Une période spécifique de l'histoire \\
\hline Marques concernées & Marques qui déclinent & Marques iconiques du passé \\
\hline Lien avec le passé & Continu & Discontinu \\
\hline Enjeu perceptuel & Crédibilité & Authenticité \\
\hline Émotion visée & Aucune spécifiquement & Nostalgie \\
\hline Exemples & $\begin{array}{l}\text { Burberry, Lacoste, Mountain } \\
\text { Dew, Harley-Davidson }\end{array}$ & $\begin{array}{l}\text { Mini, Fiat 500, } \\
\text { Led Zeppelin, Elvis Presley }\end{array}$ \\
\hline
\end{tabular}

Tableau 1 - Comparaison des stratégies de revitalisation de la marque et de rétromarquage Source : Adapté de Hallegatte (2014)

Une stratégie de revitalisation de la marque consiste généralement à s'appuyer sur l'histoire de la marque spécifiquement pour démontrer sa pertinence dans le présent. Le rétromarquage, quant à lui, consiste à s'appuyer explicitement sur une période historique dans son ensemble, comme les années 1960 pour la Fiat 500 ou les années 1970 pour Led Zeppelin. Il s'agit de vendre l'Histoire avec un grand $« \mathrm{H} »$, non pas seulement de s'appuyer sur l'histoire de la marque. 
En outre, la revitalisation s'applique à des marques qui déclinent, alors que le rétromarquage s'applique à des marques iconiques du passé qui ont été retirées du marché, habituellement sans avoir subi de déclin. Et si déclin il y a eu, ce n'est pas la raison pour laquelle la stratégie est enclenchée. Ainsi, les marques qui doivent être revitalisées ont un lien continu avec le passé, puisqu'elles n'ont pas été retirées du marché. Les marques candidates ou rétromarquage, quant à elles, ont un lien discontinu avec le passé, puisqu'elles ont été absentes du marché pendant des années. C'est justement cette absence qui les situe automatiquement dans le passé, association positive qu'il ne s'agit pas de faire disparaittre.

Par ailleurs, l'enjeu, pour les marques à revitaliser, est d'être perçues comme crédibles durant leur processus de revitalisation. Les vêtements Burberry et Lacoste seraient de nouveau tendance? La boisson Mountain Dew serait redevenue cool? Les motos Harley Davidson réincarneraient de nouveau la liberté farouche? Dans le cas du rétromarquage, l'enjeu perceptuel est plutôt celui de l'authenticité, c'est-à-dire, pour les produits remis sur le marché, de la ressemblance avec le passé.

Finalement, si dans le cas de la revitalisation de la marque, toutes sortes d'émotions peuvent être provoquées durant la mise en œuvre du marketing mix, le rétromarquage vise spécifiquement à susciter la nostalgie. En effet, nous savons qu'il s'agit d'une émotion fort positive, tout autant pour le bien-être des individus que pour les objectifs de l'organisation, tel qu'indiqué précédemment. Ainsi, le rétromarquage est une innovation radicale qui ne consiste pas à vendre de la nouveauté, mais à vendre le passé.

\section{Du vinyle au $\mathrm{mp} 3$ au vinyle}

Le retour en grâce du passé nécessitera peut-être un changement de paradigme pour le marketing des organisations. En effet, le renouvellement rapide des produits qui a nourri l'économie de marché depuis le début, et qui a été poussé jusqu'à l'absurde avec l'obsolescence programmée, la fille indigne de l'innovation, pourrait ne plus fonctionner aussi facilement qu'avant. Les consommateurs, de plus en plus informés et éduqués, ont réalisé qu'il y a un prix à payer pour le progrès, notamment celui de voir disparaitre des produits auxquels ils sont attachés, et cela pour des bénéfices douteux. En outre,

Revue Organisations \& Territoires $\bullet$ Volume $27 \bullet \mathrm{N}^{\circ} 1 \bullet 2018$ il devient de plus en plus difficile de les convaincre qu'un produit est supérieur parce qu'il est nouveau.

Le vinyle est un cas exemplaire de notre rapport dorénavant schizophrène au progrès, à l'innovation, et à la technologie. Dans les années 1980, l'industrie phonographique ne s'est pas demandé si les consommateurs avaient besoin du numérique, mais a vu dans le CD une formidable occasion de réédition, et donc de profit. Grâce à un marketing hors pair, basé sur une croyance indéfectible dans le progrès, typique des années 1980, et à un sabotage symbolique du disque vinyle, l'industrie a réussi à mettre au ban de la modernité un objet considéré seulement quelques années plus tôt comme à la fine pointe de la technologie.

A contrario, aujourd'hui, le discours selon lequel le vinyle offre un meilleur son que n'importe quel format numérique, y compris le $\mathrm{CD}$, a pris beaucoup de vigueur. Certes, chacun peut avoir son opinion sur le sujet, c'est une question de perception. Mais il est tout de même ahurissant qu'après nous avoir incité à nous débarrasser de nos disques vinyle, l'industrie nous les revende, et plus cher. Par sa stratégie de prix élevés, et d'autres éléments du marketing mix, l'industrie entérine la supériorité d'un format qu'elle avait elle-même éliminé.

Néanmoins, il ne faut pas faire correspondre l'achat d'un produit associé au passé avec une pulsion nostalgique, c'est-à-dire que ce qui appartient au passé et que le progrès a mis en obsolescence possède un intérêt intrinsèque au-delà du besoin de vivre des expériences nostalgiques. Le passé n'est pas la nostalgie et vice-versa. Pour reprendre l'exemple du vinyle, il offre une expérience sensorielle impliquant quatre des cinq sens, notamment la vue de la pochette et du livret, souvent une œuvre d'art à part entière. Son utilisation est une sorte de rituel, nécessitant de prendre soin d'un objet anthropomorphisé. Le vinyle est chargé d'associations culturelles, dont celles au rock progressif et à l'albumconcept des années 1970, aux musiques urbaines des années 1980 et au monde des DJ. A contrario, le fichier mp3 rime avec pauvreté sémiotique et privation sensorielle. Ainsi, quand bien même la nostalgie redeviendrait honnie, des vieux produits demeureront dans nos vies pour leurs qualités intrinsèques.

Cependant, rien n'est moins sûr que ce retour en grâce du passé, avec ou sans nostalgie, sonne le glas du 
dogme de l'innovation telle qu'on la connait aujourd'hui, parce que l'idée de progrès, sur lequel elle s'appuie, demeure résiliente. En effet, comme toute croyance, celle dans le progrès est basée sur un désir, en l'occurrence celui de profiter toujours plus des délices de la consommation, et de croire que les promesses de la société de consommation non remplies hier le seront demain. Comme ce désir profond est entretenu par la société de consommation en général et que, en particulier, la croyance dans le progrès est nourrie par, et nourrit, l'innovation, on peut y voir un cercle, vertueux ou vicieux selon le point de vue, où l'innovation s'autoalimente.

\section{Conclusion}

La visée de cet article est réflexive, non normative et encore moins prescriptive. Il invite à jeter un regard neuf sur le passé et la nostalgie, et à considérer par conséquent l'innovation de manière plus critique. Il serait cependant déraisonnable de la rejeter in toto. Je suggère plutôt de voir l'innovation comme contingente à une adhésion des consommateurs, laquelle n'est plus automatique, et surtout d'admettre que, par leur volonté constante d'innovation, les organisations changent la vie, mais pas forcément pour le mieux.

Il s'agit, pour les organisations, de s'adapter à ce changement profond de notre rapport collectif au passé, par pragmatisme commercial, mais aussi par souci de pertinence socioculturelle. Tenir pour acquis que les consommateurs souhaitent ardemment se voir constamment proposer des innovations serait une grossière erreur. Ils souhaitent d'abord donner un sens à leur vie, y compris dans la consommation, puisque la société les y invite. Or, les objets du passé récent, qui sont justement chargés de sens, leur sont fort utiles pour cela.

La nostalgie n'étant plus ce qu'elle était, le fait de faire référence au passé dans une publicité, ou de carrément vendre un produit associé au passé, constitue actuellement une stratégie viable. Le phénomène rétro constitue une occasion pour toute organisation capable de saisir la profondeur du changement du rapport de la société au passé. Les organisations qui sont nostalgiques de l'ancienne connotation négative de la nostalgie risquent de passer à côté d'occasions d'affaires et pire, à côté de leur époque.

Nous devons accepter l'idée que nous vivons dans une époque rétro. Il est d'ailleurs intéressant de noter que le terme « rétro » est entré dans le langage courant dans les années 1960, lorsque des rétrofusées ont été intégrées à des véhicules spatiaux, afin de créer une poussée inverse et ainsi les faire décélérer lorsqu'ils retournent dans l'atmosphère terrestre et ce, pour éviter qu'ils ne se désintègrent (Guffey, 2006). Les humains, quant à eux, achètent des produits rétro, qui constituent une force inverse à l'innovation, afin de ralentir symboliquement la marche du progrès, et ce, pour ne pas se désintégrer dans la modernité.

Il faudrait vivre dans le passé pour refuser d'admettre cela.

\section{NOTES}

1 Source : Billboard et Pollstar, compilé par AXS.com. Repéré à https://www.axs.com/top-10-highest-cash-grossing-concert-tours34634

\section{RÉFÉRENCES}

Brown, S. (2001). Marketing - The retro revolution. London, UK: Sage Publications.

Brown, S., Kozinets, R. V. et Sherry Jr., J. F. (2003). Teaching old brands new tricks: retro branding and the revival of brand meaning. Journal of Marketing, 67(3), 19-33.

Davis, F. (1979). Yearning for yesterday: a sociology of nostalgia. New York, NY: Free Press.

Fukuyama, F. (1992). The end of History and the last man. New York, NY: Free Press.

Guffey, E. E. (2006). Retro: the culture of revival. London, UK: Reaktion Books.

Hallegatte, D. (2014). Retrobranding: selling the past. Dans G. Hunter et T. Steenburgh (dir.), AMA Winter Educators' Conference Proceedings (vol. 25, p. K10-17). Orlando, FL: American Marketing Association. 
Havlena, W. J., et Holak, S. L. (1991). "The good old days": observations on nostalgia and its role in consumer behavior. Dans

R. H. Holman et M. R. Solomon (dir.), Advances in Consumer Research (vol. 18, p. 323-329). Provo, UT: Association for Consumer Research.

Keller, K. L. (2013). Strategic brand management: building, measuring, and managing brand equity. Upper Saddle River, N.J.: Pearson.

Muehling, D. D. et Pascal, V. J. (2011). An empirical investigation of the differential effects of personal, historical, and nonnostalgic advertising on consumer responses. Journal of Advertising, 40(2), 107-122.

Wildschut, T., Sedikides, C., Routledge, C., Arndt, J. et Cordaro, F. (2010). Nostalgia as a repository of social connectedness: the role of attachment-related avoidance. Journal of Personality and Social Psychology, 98(4), 573-586. 
Centre de recherche sur le développement territorial

\section{Centre de recherche sur le développement territorial}

UQAC - UQAR - UQAT - UQO - INRS-UCS - ÉNAP

Le Centre de recherche sur le développement territorial (CRDT) est un regroupement stratégique de chercheurs reconnu par le Fonds québécois de recherche sur la société et la culture.

Rassemblant près d'une cinquantaine de membres, dont une dizaine de l'extérieur du Canada, le CRDT est principalement rattaché à des institutions universitaires implantées au coeur même des régions du Québec.

Le CRDT met en oeuvre une programmation intégrée de recherche comparative sur le développement territorial :

\section{AXE 1}

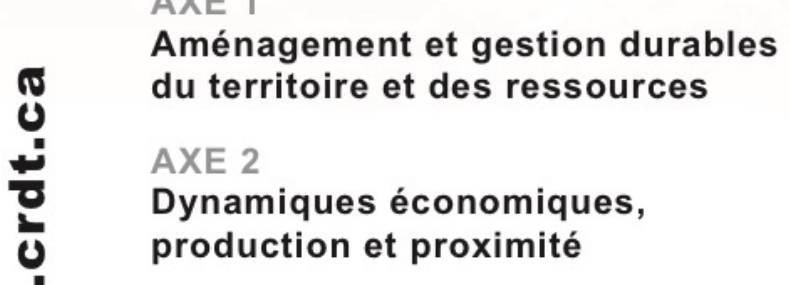

AXE 3

Politiques publiques et gouvernance territoriale
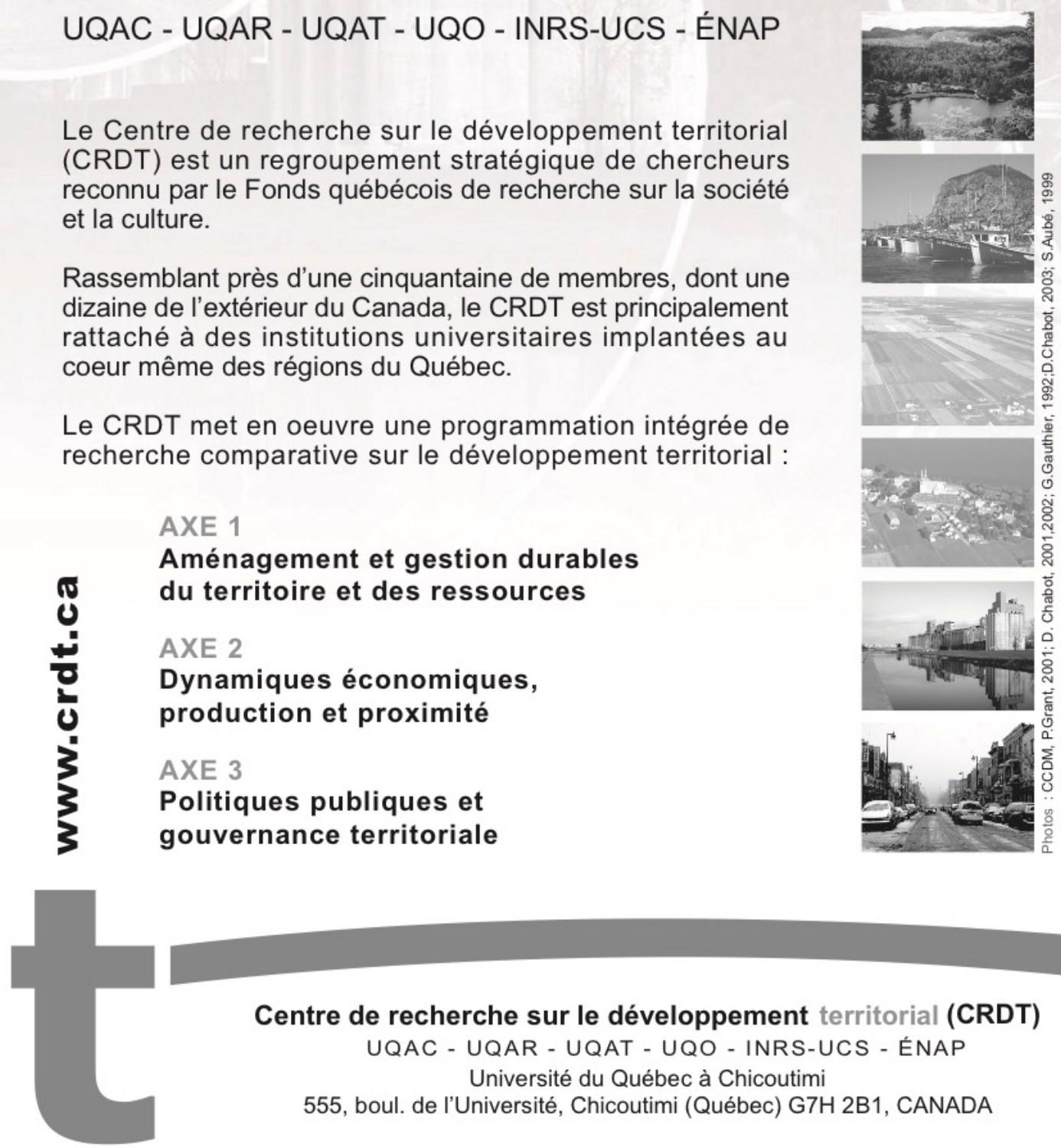

Centre de recherche sur le développement territorial (CRDT)

UQAC - UQAR - UQAT - UQO - INRS-UCS - ÉNAP

Université du Québec à Chicoutimi

555, boul. de l'Université, Chicoutimi (Québec) G7H 2B1, CANADA 